\title{
Di-Phenyl Ether Derivatives as a Potential Liquid Organic Hydrogen Carriers: Thermochemical and Computational study
}

Sergey P. Verevkin, ${ }^{a, b, c, *}$ Riko Siewert, ${ }^{a, b}$ Vladimir N. Emel'yanenko, ${ }^{c}$ Jason E. Bara, ${ }^{d}$ Hongda Cao, ${ }^{\mathrm{e}}$ Andrey A. Pimerzin ${ }^{c}$

aDepartment of Physical Chemistry, University of Rostock, 18059, Rostock, Germany

${ }^{b}$ Faculty of Interdisciplinary Research, Competence Centre CALOR, University of Rostock, 18051 Rostock, Germany

${ }^{c}$ Chemical Department, Samara State Technical University, 443100, Russia

${ }^{d}$ University of Alabama, Department of Chemical \& Biological Engineering, Tuscaloosa, AL USA 35487-0203

${ }^{e}$ University of Alabama, Department of Chemistry \& Biochemistry, Tuscaloosa, AL USA 354870336

${ }^{*}$ Corresponding author.

E-mail address: sergey.verevkin@uni-rostock.de (S.P. Verevkin)

Combustion Calorimetry., ${ }^{1,2}$ The liquid samples were encapsulated in small polyethylene ampoules. The solid samples were pressed into pellets. Small polyethylene (PE) pieces were used as an auxiliary material in order to reach complete combustion. The pellet was placed in the crucible, surrounded with PE pieces and was burned in $\mathrm{O}_{2}$ at a pressure of $3.04 \mathrm{MPa}$. All weightings were performed by using a microbalance with $10^{-6} \mathrm{~g}$ resolution. The bomb was not prepurged with $\mathrm{O}_{2}$. Test experiments with and without purging with $\mathrm{O}_{2}$ showed results consistent within the experimental uncertainty. The combustion products were examined for carbon monoxide ( $\mathrm{CO}$ ) (Dräger tube) and unburned carbon, but neither was detected. The energy equivalent of the calorimeter $\varepsilon_{\text {calor }}$ was determined with a standard reference sample of benzoic acid (sample SRM 39j, NIST). Correction for nitric acid $\left(\mathrm{HNO}_{3}\right)$ formation was based on titration with $0.1 \mathrm{~mol} \cdot \mathrm{dm}^{-3} \mathrm{NaOH}(\mathrm{aq})$. Auxiliary data required for the reduction are presented in Table S1.

Table S1. Formula, Density $\rho(T=293 \mathrm{~K})$, Specific Heat Capacity $c_{\mathrm{p}}(T=298.15 \mathrm{~K})$, and Expansion Coefficients $(\delta V / \delta T)_{\mathrm{p}}$ of the Materials Used in the Present Study

\begin{tabular}{|c|c|c|c|c|}
\hline \multirow{2}{*}{ Compounds } & \multirow{2}{*}{ formula } & $\rho$ & $c_{\mathrm{p}}{ }^{\mathrm{a}}$ & $10^{-6} \cdot(\delta V / \delta T)_{p}$ \\
\hline & & $\mathrm{g} \cdot \mathrm{cm}^{-3}$ & $\mathrm{~J} \cdot \mathrm{K}^{-1} \cdot \mathrm{g}^{-1}$ & $\mathrm{dm}^{3} \cdot \mathrm{K}^{-1}$ \\
\hline 3-methyl-diphenyl ether & $\mathrm{C}_{13} \mathrm{H}_{12} \mathrm{O}$ & $1.051[3]$ & 1.67 & 1.0 \\
\hline 3-methoxy-diphenyl ether & $\mathrm{C}_{13} \mathrm{H}_{12} \mathrm{O}_{2}$ & $1.116[4]$ & 1.66 & 1.0 \\
\hline 2-hydroxy-diphenyl ether & $\mathrm{C}_{12} \mathrm{H}_{10} \mathrm{O}_{2}$ & $1.17[5]$ & 1.20 & 0.1 \\
\hline polyethylene $^{\mathrm{b}}$ & $\mathrm{CH}_{1.93}$ & 0.92 & 2.53 & 0.1 \\
\hline cotton $\mathrm{b}$ & $\mathrm{CH}_{1.774} \mathrm{O}_{0.887}$ & 1.50 & 1.67 & 0.1 \\
\hline
\end{tabular}

${ }^{a}$ Estimated by group-contribution method (see Table S2)

$\mathrm{b}$ Data for density, specific heat capacity, and expansion coefficients of auxiliary materials are from our previous work [6]: energy of combustion $\Delta_{\mathrm{c}} u^{\circ}$ (polyethylene) $=-46357.3 \mathrm{~J} \cdot \mathrm{g}^{-1} ; u\left(\Delta_{\mathrm{c}} u^{\circ}\right)=$ $3.6 \mathrm{~J} \cdot \mathrm{g}^{-1}$; energy of combustion $\Delta_{\mathrm{c}} u^{\circ}($ cotton $)=-16945.2 \mathrm{~J} \cdot \mathrm{g}^{-1} ; u\left(\Delta_{\mathrm{c}} u^{\circ}\right)=4.2 \mathrm{~J} \cdot \mathrm{g}^{-1}$. 
Transpiration Method. ${ }^{7-9}$ Vapor pressures of diphenyl ether derivatives were measured using the transpiration method. About $0.5 \mathrm{~g}$ of the sample was mixed with small glass beads and placed in the thermostatted U-shaped saturator. A stream of $\mathrm{N}_{2}$ with a well-defined flow rate was passed through the saturator at constant temperature $( \pm 0.1 \mathrm{~K})$, and the transported material was collected in a cold trap. The amount of condensed substance was determined by GC. The saturation vapor pressure $p_{\text {sat }}$ at each temperature $T_{i}$ was calculated from the amount of product collected within a definite period of time:

$$
p_{i}=m_{i} \cdot R \cdot T_{\mathrm{a}} / V \cdot M_{i} ; \quad V=\left(n_{\mathrm{N} 2}+n_{i}\right) \cdot R \cdot T_{\mathrm{a}} / P_{\mathrm{a}}
$$

where $V$ is the volume of the gas phase consisting of the $n_{\mathrm{N} 2}$ moles of the carrier gas and $n_{i}$ mole of gaseous compound under study at the atmospheric pressure $P_{\mathrm{a}}$ and the ambient temperature $T_{\mathrm{a}}$. The $\mathrm{N}_{2}$ gas flow rate was measured with the HP soap film flowmeter (model 0101-0113). The amount of the carrier gas $n_{\mathrm{N} 2}$ was determined from the flow rate and time. The amount of the compound under study $n_{i}$ in the carrier gas was estimated applying the ideal gas law at each temperature. Prior to the beginning the vapour pressure measurements, the pre-conditioning of the sample was performed first at (293-300) K (two hours) in order to withdraw possible water traces. Then the saturator was kept at 320-350 K (in order to remove possible traces of volatile compounds). In order to ascertain the completing of pre-conditioning, three samples were taken during the sample flashing at a suitable temperature and analysed by the GC. A constant vapour pressure at this temperature indicated that the transpiration experiments could start. No additional impurities were detected by the GC analysis of the transported material. The absence of impurities and decomposition products was rechecked by GC analysis of the saturator content after completing of the whole series of experiments.

Differential Scanning Calorimetry (DSC). The thermal behavior of the crystalline samples including melting temperature and enthalpy of fusion were studied with a commercial DSC Mettler Toledo DSC 822e coupled with Huber TC100MT cooler. The DSC was standardized with melting behavior of the reference compounds indium, tin, lead, water, and $n$-octane. Samples of about 25 $\mathrm{mg}$ were weighed with Sartorius MSE3.6P-000-DM microbalances with a resolution of $5 \cdot 10^{-6} \mathrm{~g}$, hermetically sealed in standard non-pinned aluminum pans of $40 \mu \mathrm{L}$ volume. Sample was loaded in the pan and preliminary heated to $20 \mathrm{~K}$ above the melting temperature and then cooled to 193 K. Such a procedure served to obtain a sufficient thermal contact between the sample and the bottom of the pan. After that, the pan was consequently heated and cooled a few times in the temperature range of $193 \mathrm{~K}$ to the melting temperature at rates of 10 and $50 \mathrm{~K} \cdot \mathrm{min}^{-1}$. From our experience, such a sequence of heating/cooling rates allows for detection of a possible polymorphic transitions or coexisting phases in the sample under study. The DSC measurements 
were repeated in triplicate. The melting temperatures were evaluated as the onset temperature of observed transition adjusted to the zero-heating rate. The uncertainties of the temperature and enthalpy of fusion are expressed as extended uncertainties with confidence level of $95 \%(\mathrm{k} \approx 2)$.

Table S2. Compilation of Data on Molar Heat Capacities $C_{\mathrm{p}, \mathrm{m}}^{\mathrm{o}}$ and Differences $\Delta_{\mathrm{l}, \mathrm{cr}}^{\mathrm{g}} C_{\mathrm{p}, \mathrm{m}}^{\mathrm{o}}$ of

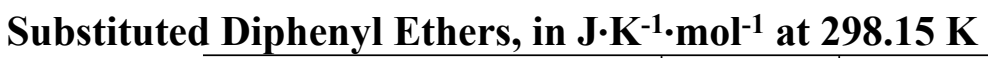

\begin{tabular}{l|ccccc}
\hline compound & $C_{\mathrm{p}, \mathrm{m}}^{\mathrm{o}}(\mathrm{cr})^{\mathrm{a}}$ & $-\Delta_{\mathrm{cr}}^{\mathrm{g}} C_{\mathrm{p}, \mathrm{m}}^{\mathrm{o}} \mathrm{C}_{\mathrm{p}, \mathrm{m}}^{\mathrm{o}}(\mathrm{liq})^{\mathrm{a}}$ & $-\Delta_{\mathrm{p}}^{\mathrm{g}} C_{\mathrm{p}, \mathrm{m}}^{\mathrm{o}}$ \\
\hline 3-methyl-diphenyl ether & 238.1 & 36.5 & 305.0 & 89.9 \\
3-methoxy-diphenyl ether & 256.6 & 39.2 & 333.0 & 97.2 \\
2-hydroxy-diphenyl ether & 224.2 & 34.4 & 328.8 & 96.1 \\
4-hydroxy-diphenyl ether & 224.2 & 34.4 & 328.8 & 96.1 \\
\hline
\end{tabular}

${ }^{a}$ Calculated according to the additive procedure developed by Chickos et al. ${ }^{10}$

${ }^{\mathrm{b}}$ Calculated according to the procedure developed by Acree and Chickos. ${ }^{11}$

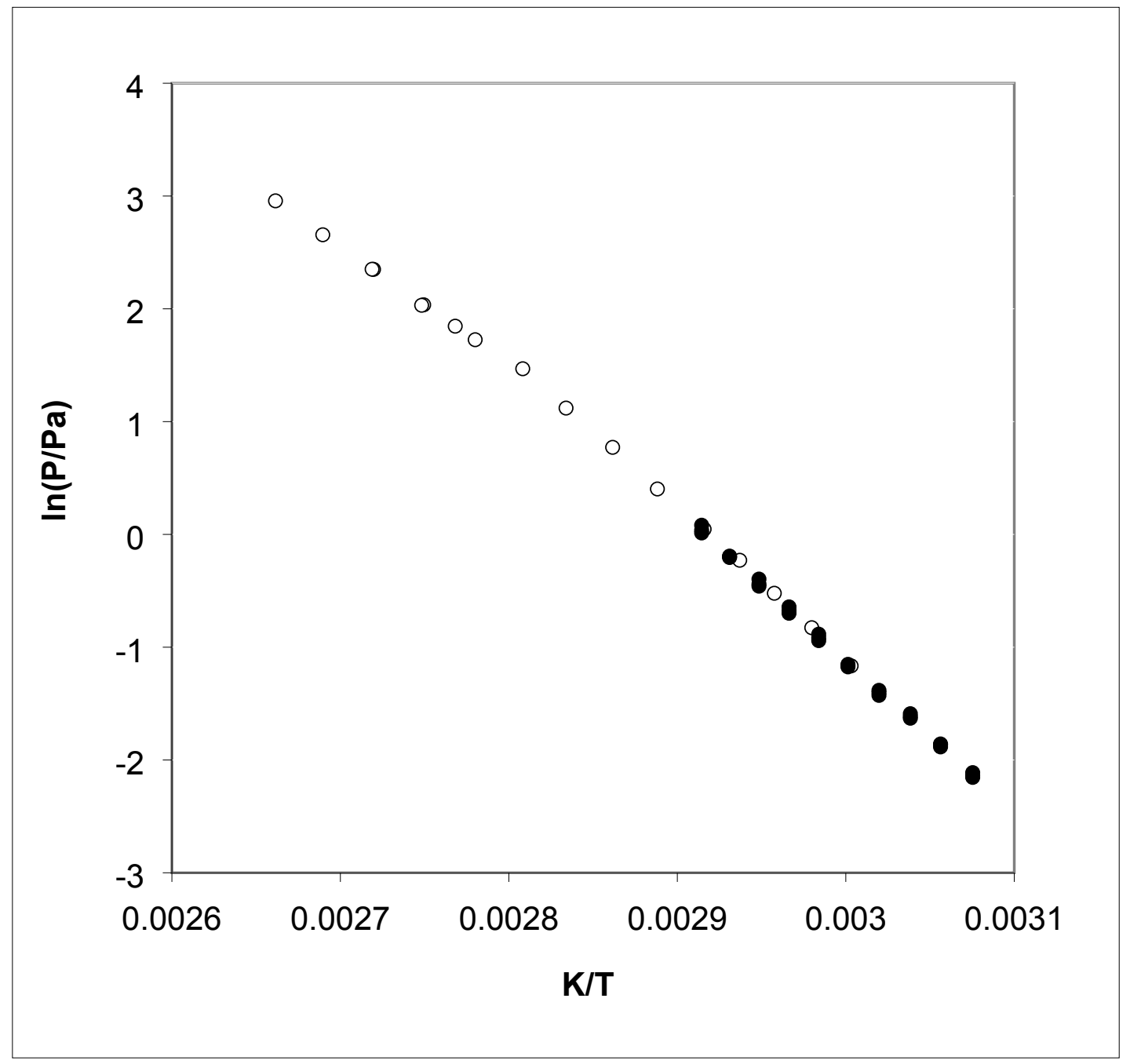

Figure S1. Temperature dependence of vapor pressures over the solid and liquid 4-hydroxydiphenyl ether: $\bigcirc$ - this work, transpiration; • - ref. [5], Knudsen method. 
Table S3. Gas-Phase Standard Molar Thermodynamic Properties of Benzene Derivatives and Their Fully Hydrogenated Analogues at $T=298.15 \mathrm{~K}$

\begin{tabular}{|c|c|c|c|c|}
\hline compound & $\Delta_{\mathrm{f}} H_{\mathrm{m}}^{\mathrm{o}}(\mathrm{g})^{\mathrm{a}}$ & Ref. & $S_{\mathrm{m}}^{\mathrm{o}}(\mathrm{g})$ & Ref. \\
\hline & $\mathrm{kJ} \cdot \mathrm{mol}^{-1}$ & & $\mathrm{~J} \cdot \mathrm{K}^{-1} \cdot \mathrm{mol}^{-1}$ & \\
\hline benzene & $82.6 \pm 0.7$ & 12 & 269.2 & 14 \\
\hline cyclohexane & $-123.4 \pm 0.8$ & 12 & 298.2 & 14 \\
\hline diphenyl ether & $50.9 \pm 1.4$ & 13 & 426.0 & 13 \\
\hline dicyclohexyl-ether & $-360 \pm 4.5$ & 13 & 471.7 & 15 \\
\hline methyl-benzene & $50.4 \pm 0.6$ & 12 & 320.7 & 14 \\
\hline methyl-cyclohexane & $-154.7 \pm 1.0$ & 12 & 343.3 & 14 \\
\hline 3-methyl-diphenyl ether & $16.0 \pm 3.6$ & Table 5 & 469.4 & 15 \\
\hline 3-methyl- dicyclohexyl-ether & $-389.1 \pm 5.0$ & $\mathrm{GA}^{\mathrm{b}}$ & 506.2 & 15 \\
\hline methoxy-benzene & $-70.7 \pm 1.4$ & 16 & 354.5 & 14 \\
\hline methyl-cyclohexyl ether & $-271.8 \pm 5.0$ & $\mathrm{GA}^{\mathrm{b}}$ & 377.8 & 15 \\
\hline 3-methoxy-diphenyl ether & $-105.4 \pm 4.2$ & Table 5 & 490.6 & 15 \\
\hline 3-methoxy-dicyclohexyl-ether & $-504.8 \pm 5.0$ & $\mathrm{GA}^{\mathrm{b}}$ & 536.4 & 15 \\
\hline hydroxy-benzene & $-96.4 \pm 0.9$ & 12 & 315.6 & 14 \\
\hline cyclohexanol & $-286.2 \pm 2.1$ & 17 & 353.8 & 17 \\
\hline 4-hydroxy-diphenyl ether & $-126.5 \pm 2.9$ & Table 5 & 455.5 & 15 \\
\hline 4-hydroxy-cyclohexyl-cyclohexane & $-526.0 \pm 5.0$ & $\mathrm{GA}^{\mathrm{b}}$ & 511.0 & 15 \\
\hline
\end{tabular}

a The uncertainties of enthalpies of formation are presented as expanded uncertainty with 0.95 level of confidence.

${ }^{\mathrm{b}} \mathrm{GA}=$ calculated using group-contributions derived in our previous work [18-20].

\section{References}

(1) Verevkin, S. P.; Schick, C. Substituent Effects on the Benzene Ring. Determination of the Intramolecular Interactions of Substituents in tert-Alkyl-Substituted Catechols from Thermochemical Measurements. J. Chem. Eng. Data 2000, 45, 946-952.

(2) Emel'yanenko, V. N.; Verevkin, S. P.; Heintz, A. The gaseous enthalpy of formation of the ionic liquid 1-butyl-3-methylimidazolium dicyanamide from combustion calorimetry, vapor pressure measurements, and ab initio calculations. J. Am. Chem. Soc. 2007, 129.

(3) Kranzfelder, A. L.; Verbanc, J. J.; F. J. Sowa, F. J. The Cleavage of Diphenyl Ethers by Sodium in Liquid Ammonia. II. Meta Substituted Diphenyl Ethers. J. Am. Chem. Soc. 1937, 59, 14881490.

(4) Suter, C. M.; Green, F. O. Phenoxthin. II. Extension of the Ferrario Reaction. J. Am. Chem. Soc. 1937, 59, 2578-2580.

(5) Ribeiro da Silva, M. A. V.; Lobo Ferreira, A. I. M. C.; Cimas, Á. Calorimetric and Computational Study of the Thermochemistry of Phenoxyphenols J. Org. Chem. 2011, 76 37543764.

(6) Verevkin, S. P. Thermochemistry of phenols: quantification of the ortho-, para-, and metainteractions in tert-alkyl substituted phenols, J. Chem. Thermodyn. 1999, 31, 559-585.

(7) Verevkin, S. P.; Emel'yanenko, V. N. Transpiration method: Vapor pressures and enthalpies of vaporization of some low-boiling esters. Fluid Phase Equil. 2008, 266, 64-75. 
(8) Verevkin, S. P.; Sazonova, A. Y.; Emel'yanenko, V. N.; Zaitsau, D. H.; Varfolomeev, M. A.; Solomonov, B. N.; Zherikova, K. V. Thermochemistry of Halogen-Substituted Methylbenzenes. J. Chem. Eng. Data 2014, 60, 89-103.

(9) Emel'yanenko, V. N.; Verevkin, S. P. Benchmark thermodynamic properties of 1,3propanediol: Comprehensive experimental and theoretical study. J. Chem. Thermodyn. 2015, 85, 111-119.

(10) Chickos, J. S.; Hosseini, S.; Hesse, D. G.; Liebman, J. F. Heat capacity corrections to a standard state: a comparison of new and some literature methods for organic liquids and solids. Struct. Chem. 1993, 4, 271-278.

(11) Acree, W.; Chickos, J. S. Phase Transition Enthalpy Measurements of Organic and Organometallic Compounds and Ionic Liquids. Sublimation, Vaporization, and Fusion Enthalpies from 1880 to 2015. Part 2. C 11 -C 192. J. Phys. Chem. Ref. Data 2017, 46, 13104.

(12) Pedley, J.B.; Naylor, R.D.; Kirby, S.P., Thermochemical Data of Organic Compounds, Chapman and Hall, New York, 1986, 1-792.

(13) Emel'yanenko, V. N.; Zaitsau, D. H.; Pimerzin, A. A.; Verevkin, S. P. Benchmark properties of diphenyl oxide as a potential liquid organic hydrogen carrier: Evaluation of thermochemical data with complementary experimental and computational methods. J. Chem. Thermodyn. 2018, $125,149-158$

(14) Stull D.R., Jr., The Chemical Thermodynamics of Organic Compounds. Wiley, New York, 1969.

(15) Domalski, E.S.; Hearing, E.D., Estimation of Thermodynamic Properties of C-H-N-OHalogen Compounds at 298.15 K, J. Phys. Chem. Ref. Data 1993, 22, 805-1159.

(16) Simões, R. G.; Agapito, F.; Diogo,H. P.; Minas da Piedade, M. E. The Enthalpy of Formation of Anisole: Implications for the Controversy on the O-H Bond Dissociation Enthalpy in Phenol, J. Phys. Chem. A 2014 118, 11026-11032

(17) Kabo G.J., Thermodynamic properties of cyclohexanol and cyclohexanone, J. Chem. Thermodyn. 1988, 20, 429-437.

(18) Verevkin, S. P.; Emel'yanenko, V. N.; Diky, V.; Muzny, C. D.; Chirico, R. D.; Frenkel, M. New Group-Contribution Approach to Thermochemical Properties of Organic Compounds: Hydrocarbons and Oxygen-Containing Compounds. J. Phys. Chem. Ref. Data 2013, 42, 33102.

(19) Roganov, G. N.; Pisarev, P. N.; Emel'yanenko, V. N.; Verevkin, S. P. Measurement and Prediction of Thermochemical Properties. Improved Benson-Type Increments for the Estimation of Enthalpies of Vaporization and Standard Enthalpies of Formation of Aliphatic Alcohols. $J$. Chem. Eng. Data 2005, 50, 1114-1124.

(20) Verevkin, S. P. Improved Benson`s Increments for the Estimation of the Standard Enthalpies of Formation and Enthalpies of Vaporization of Alkyl Ethers, Acetals, Ketals, and Orthoesters. $J$. Chem. Eng. Data 2002, 47, 1071-1097. 